\title{
Time-Resolved Cathodoluminescence in an Ultrafast Transmission Electron Microscope
}

\author{
S. Meuret*1, L. H.G. Tizei ${ }^{2}$, F. Houdellier ${ }^{1}$, S. Weber ${ }^{1}$, Y. Auad ${ }^{2}$, M. Tencé2 ${ }^{2}$ H.-C. Chang ${ }^{3}$, M. Kociak² and A. \\ Arbouet*1
}

1 - CEMES-CNRS Université de Toulouse 29 rue Jeanne Marvig 31055 Toulouse France

2 - Université Paris-Saclay, CNRS, Laboratoire de Physique des Solides, 91405, Orsay, France

3 - Institute of Atomic and Molecular Sciences, Academia Sinica, Taipei 106, Taiwan

Authors to whom correspondence should be addressed : [Sophie Meuret, Sophie.meuret@cemes.fr; Arnaud Arbouet : arnaud.arbouet@cemes.fr]

\begin{abstract}
Ultrafast transmission electron microscopy (UTEM) combines sub-picosecond time-resolution with the versatility of TEM spectroscopies. It allows to study the ultrafast materials response using complementary techniques. However, until now, time-resolved cathodoluminescence was unavailable in a UTEM. In this paper we report time-resolved cathodoluminescence measurements in an ultrafast transmission electron microscope. We mapped the spatial variations of the emission dynamics from nano-diamonds with a high density of NV centers, with a $12 \mathrm{~nm}$ spatial resolution and sub-nanosecond temporal resolution. This development will allow to study the emission dynamics from quantum emitters with a unique spatiotemporal resolution and benefit from the wealth of complementary signals provided by transmission electron microscopes. It will further expand the possibilities of ultrafast Transmission Electron Microscopes paving the way to the investigation of the quantum aspects of electron/sample interaction.
\end{abstract}

Excited states in atomic, molecular or solid-state systems can decay through a great variety of nonradiative or radiative relaxation channels. Light emission, also called luminescence, associated with radiative pathways contains invaluable information on the properties of these systems and their dynamics. Measuring the lifetime of an excited state is therefore essential to understand complex relaxation pathways. The study of light emission has motivated over the course of history a large number of major instrumental developments. The developed techniques have long relied on optical excitation of the sample ${ }^{1-7}$. However, the limited spatial resolution of optical spectroscopies and the sub-wavelength length scale of several important relaxation processes stimulated the search for alternative strategies. The excellent spatial resolution of electron microscopes and the richness of the secondary signals produced by the interaction between fast electrons and matter motivated very early ambitious developments to obtain with the same instrument information on the chemical structure (X-ray), surface properties (secondary electrons) and optical properties ((near-)visible light). One of the advantages of Transmission Electron Microscopy (TEM) over Scanning Electron Microscopy (SEM) is the possibility to study the electron after its interaction with the sample. This allows for instance to analyze the electron beam energy exchange with the material (electron energy loss spectroscopy: EELS), or to determine the crystallographic structure of the sample (diffraction imaging) as well as to quantitatively map the electric and magnetic fields (Electron holography). TEM is therefore one of the most versatile tools to study matter at the nanometer 
scale. In a TEM, information on the material optical properties can be gained through the study of the generated visible light via Cathodoluminescence $(\mathrm{CL})$ or the analysis of the EELS in the low-loss region of the spectrum, the two techniques giving access to different optical quantities ${ }^{8,9}$. The development of $\mathrm{CL}$ spectroscopy in a scanning TEM (STEM-CL) has been a major milestone ${ }^{10-12}$ that allowed the study of solid state systems structured at the atomic scale, such as Light Emitting Diodes (LEDs), semiconductor lasers or color centers ${ }^{13}$ at the relevant scale. STEM-CL was for example used to study the quantum Stark effect of III-V heterostructures ${ }^{14,15}$, to spatially resolve atomic defects in diamond ${ }^{16}$, or reveal the connection between the formation of GaN/AIN quantum dots and threading dislocations ${ }^{17}$. It has become a wellestablished technique in nano-optics. However, the continuous electron beam of standard electron microscopes has long restricted cathodoluminescence to the study of steady state optical properties and prevented the measurement of the excited state lifetime or the carrier mobility. Recently, the measurement of the $\mathrm{CL}$ autocorrelation function allowed the measurement of the lifetime even with a continuous beam ${ }^{18-20}$ but with a low signal to noise ratio (SNR) due to its dependence on the square of the intensity ${ }^{21}$. However, it demonstrated the possibility and interest of measuring lifetimes with sub 10$\mathrm{nm}$ spatial resolution even in buried structures [12].

The search for time-resolved $\mathrm{CL}$ measurement using a pulsed electron excitation was therefore sought after early. Indeed, the first time-resolved cathodoluminescence study in an SEM was performed in the 80 's. Christen and others ${ }^{22-24}$, used a blanking scheme where the electron beam was swept over an aperture ${ }^{25-27}$. Despite a long pulse ( $\left.>100 \mathrm{~ns}\right)$ the sharp falling edge $(<1 \mathrm{~ns})$ resulted in a sub-nanosecond resolution but at the expense of a limited spatial resolution $(>1 \mu \mathrm{m})$. It is only in 2005 that Merano, Sonderegger, Crottini et al ${ }^{28}$ achieved the first time-resolved cathodoluminescence study combining picosecond temporal resolution and 50 nanometer spatial resolution. They used a laser-driven gold photocathode to generate a picosecond pulsed electron beam ${ }^{29-31}$. Since then, time-resolved cathodoluminescence in a SEM has been used to study the carrier relaxation dynamics ${ }^{32}$ as well as the influence of basal stacking faults ${ }^{33}$, strain ${ }^{34}$ or exciton mobility ${ }^{35}$ on the optical properties. Until now, time-resolved cathodoluminescence studies have only been performed in SEMs, limiting the achievable spatial resolution and material properties accessible from complementary signals.

In this letter we report a time-resolved cathodoluminescence study performed in a pulsed electron gun TEM. We measured the spatial variation of the lifetime of atomic defects (nitrogen vacancy - NV - center) in nano-diamonds with a sub-nanosecond temporal resolution and $12 \mathrm{~nm}$ spatial resolution.

Figure 1-a,c shows a sketch of the experimental set-up ; an Hitachi HF2000 ultrafast transmission electron microscope (UTEM) equipped with an ultra-fast cold-field emission source described in previous articles 36-38. A femtosecond laser generates a $400 \mathrm{fs}$ pulsed electron beam from a sharp tungsten tip at a repetition rate of $2 \mathrm{MHz}$. The electrons are then accelerated at $150 \mathrm{keV}$. The pulsed electron beam can be focused on the sample within a probe of less than $1 \mathrm{~nm}$, and scanned over the region of interest ${ }^{37}$. A parabolic mirror collects the cathodoluminescence generated by the interaction of the electron beam with the sample and directs it to a multimode optical fiber. The main challenge of cathodoluminescence in a UTEM is the signal to noise ratio as the current of the pulsed electron beam (about $100 \mathrm{fA}$ on the sample ${ }^{39}$ ) is much smaller than the one generated with a conventional TEM leading to a drastically decreased CL signal. The large collection angle of the parabolic mirror is therefore essential to collect efficiently the luminescence signal. It is followed by a lens whose numerical aperture is matched to an optical fiber redirecting the $\mathrm{CL}$ signal either to an optical spectrometer or to a Single Photon Counting Module (SPCM) connected to a correlator. Using the open-source acquisition software PyMoDAQ ${ }^{40}$, we can record either 
the optical spectrum or the decay trace at each position of the electron beam. In this work, we studied nano-diamonds containing a large number of NV centers ${ }^{41,42}$ with a mean diameter of about $100 \mathrm{~nm}$. The $\mathrm{NV}$ center is an atomic defect in the diamond crystal lattice consisting of a substitutional nitrogen atom and a neighboring vacancy. It is a very well-known single photon emitter particularly stable under electron excitation and bright at room temperature ${ }^{43,44}$. The NV center has two different charge states, the neutral $\mathrm{NV}^{0}$ and the negatively charged $\mathrm{NV}^{-}$. Cathodoluminescence spectroscopy is probing almost exclusively the $\mathrm{NV}^{0}$ state ${ }^{45}$. Figure 1-b displays a typical cathodoluminescence spectrum of nano-diamonds used in this study. It was recorded with a continuous electron beam, at room temperature in a VG HB510 scanning TEM. The zero-phonon line emission is visible at $575 \mathrm{~nm}$ as well as the broad sideband associated with phonon-assisted emission from 575 to $800 \mathrm{~nm}$.

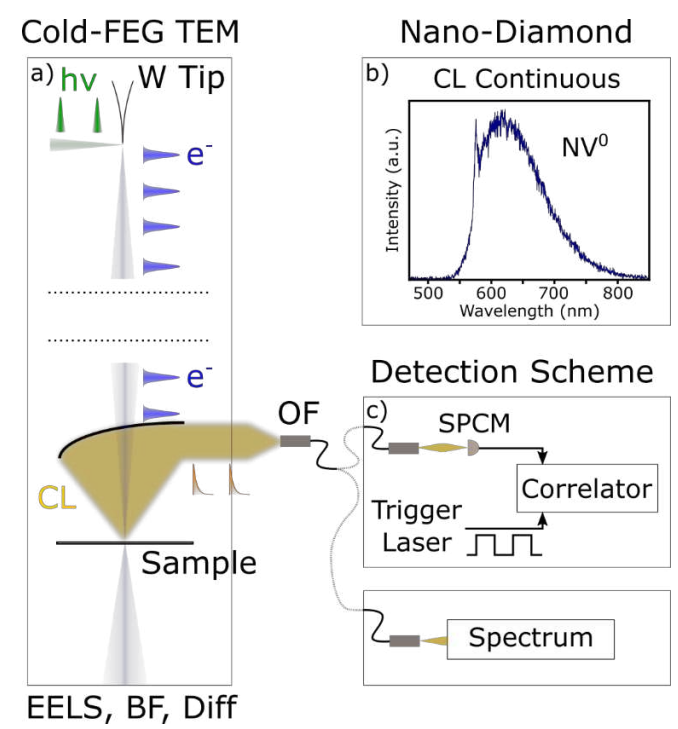

Figure 1: Schematics of the experimental set-up. a) In a modified cold-FEG Hitachi HF2000 microscope, a $2 \mathrm{MHz}$ femto-second laser at $515 \mathrm{~nm}$ triggers the emission of femtosecond pulses of electrons from a tungsten tip. A parabolic mirror collects the cathodoluminescence from the sample. c) Thanks to an optical fiber coupled to the parabolic mirror through a lens (not shown) the light goes either to a spectrometer or to a single photon counting module (SPCM). In the later, a correlator records a histogram of the delays between excitation (trigger) and $C L$ photon detection. Such a histogram forms a decay trace from which

the lifetime of the excited state can be extracted. b) Spectrum of a diamond NV center, recorded in a VG510 scanning

transmission electron. This spectrum was taken with a continuous beam at room temperature on the same batch of nanodiamonds used for this study. One can see the zero-phonon line at $575 \mathrm{~nm}$ followed by the phonon replica from 575 to $800 \mathrm{~nm}$.

Figure 2 shows the spectrum and decay trace taken for a fixed position of the electron beam on a nanodiamond cluster. In order to maximize the SNR for the acquisition of the CL spectrum (Figure 2-a), no condenser aperture was inserted in the electron column to maximize the electron beam current. This induces an increase of the electron spot size due to the geometrical aberrations of condenser lenses. The spectrum acquisition time was $30 \mathrm{~s}$. Nevertheless, we can notice that there is still enough spatial resolution to observe a change in intensity between the two different positions (about $400 \mathrm{~nm}$ apart) as noted in the 
insert of Figure 2-a. The peak at $515 \mathrm{~nm}$ visible in the spectrum is mostly due to scattering from the 515 $\mathrm{nm}$ laser exciting the tungsten tip in the electron gun. Direct excitation of the nano-diamonds by the scattered laser light can however be discarded as (i) the number of photons travelling from the tungsten tip to the sample is obviously very low due small apertures size along the electron optics (mainly source extractor and differential aperture), (ii) a significant contribution from such direct optical excitation is not consistent with the observed variations of the signal with the electron beam position and (iii) direct optical excitation of the NV centers would yield a significant contribution from the negatively charged $\mathrm{NV}^{-}$charge state and was not observed. Figure 2-b displays the decay trace measured at the same position as the blue spectrum of Figure 2-a. To acquire this decay trace, we record the delay between the excitation (trigger: electronic signal sent from the laser) and the $\mathrm{CL}$ photon detection. Due to the correlated acquisition between the excitation and the detection, the SNR improves greatly and we can add an aperture to improve the probe size down to about $5 \mathrm{~nm}$. A long path filter at $550 \mathrm{~nm}$ is added in front of the SPCM detector in order to remove the laser tail visible on the spectrum (Figure 2-a). In these conditions, we detect 40 photons (above the dark noise) per second on the APD. The decay trace displayed in Figure 2-b is taken with an integration time of $200 \mathrm{~s}$.
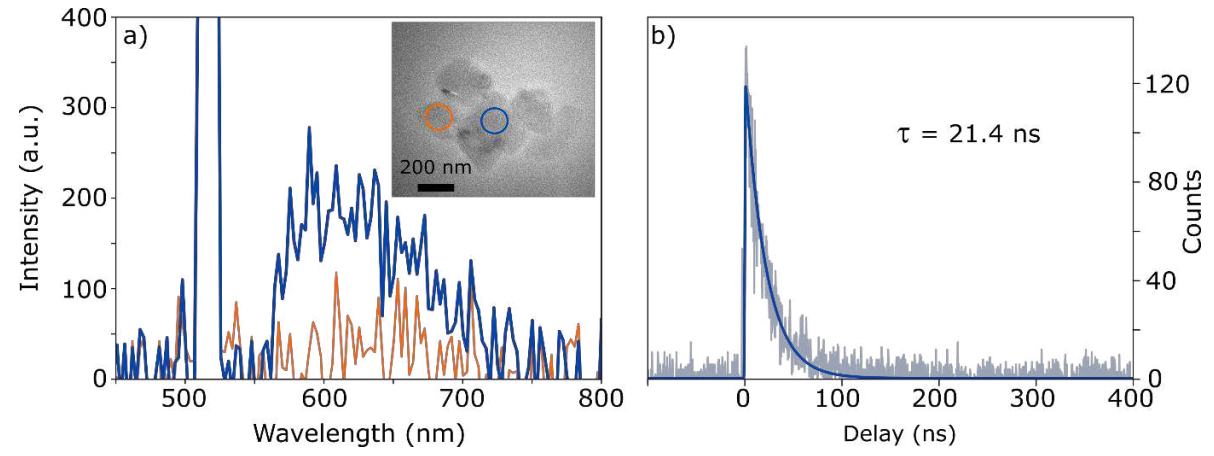

Figure 2 : Spectrum and decay trace measured in the HF2000 based UTEM a) Average spectrum of a nano-diamond cluster (TEM image in Inset). The integration time was $30 \mathrm{~s}$, and no illumination aperture was used to maximize the signal. b) Decay trace recorded for $200 \mathrm{~s}$, the electron probe (5 $\mathrm{nm}$ spot using a $70 \mu \mathrm{m}$ illumination aperture) excited the area highlighted by the blue circle in the inset of a). An exponential fit (thick blue line) gives a lifetime $\tau$ of $21.4 \mathrm{~ns}$ in the expected range for nano-diamond lifetimes.

In order to extract the lifetime from the decay trace we fit the data with the convolution of an exponential decay and a gaussian. The gaussian function accounts for the time resolution of the experiment. It could also account for a slowly increasing contribution associated to the diffusion of carriers but in the case of nano-diamonds excited by $150 \mathrm{keV}$ electrons we can safely consider the excitation of the defect centers instantaneous compared to the nanosecond lifetime. The instrument response function of the experiment is defined as IRF $=\sqrt{\sum \sigma_{i}^{2}}$ where $\sigma_{i}$ accounts for the different sources of error. In our case the contribution from the electron pulse-width (about $400 \mathrm{fs}$ ) is negligible, the time bin is set to $400 \mathrm{ps}$ and the photon detector has a resolution of $350 \mathrm{ps}$. We therefore expect an IRF of about $530 \mathrm{ps}$. In the case of Figure 2-b, the fit yields an IRF of 873 ps which is close to what we expect considering the 512 ps sampling of the 
histogram. The lifetime found for the decay trace displayed in Figure 2-b is $21.4 \pm 0.5 \mathrm{~ns}$, in the range of expected values for nano-diamonds ${ }^{46}$.

These nano-diamonds contain much more than one center. The carrier diffusion length being of the order of $50 \mathrm{~nm}{ }^{16}$, we expect that the electron beam excites all the centers whatever its position in the nanodiamond. However, the excitation strength of a given center depends on (i) its position inside the diamond and (ii) its position relative to the electron beam. The measured lifetime is therefore an average of the lifetimes of all the centers excited by the electron beam. Nevertheless, lifetime correlation-based measurements performed on similar diamonds have shown a strong disparity of the averaged lifetimes measured between objects ${ }^{47}$.

Therefore, we show in Figure 3 the results of spatially-resolved lifetime measurements performed with Time resolved STEM-CL. We scanned the electron beam over the nano-diamonds cluster and recorded a decay trace at each position. Using the same STEM aperture as for the decay trace shown in Figure 2-b (5 $\mathrm{nm}$ probe), we scanned a $200 \mathrm{~nm}$ region with square pixels of $6 \mathrm{~nm}$ width and an integration time of $5 \mathrm{~s}$ per pixel. Figure $3-b$ shows the intensity map extracted from the decay trace map by summing, for each pixel, all the histogram bins overs a $500 \mathrm{~ns}$ window ( $2 \mathrm{MHz}$ repetition rate). We see quite clearly the intensity variations over the scan depending on the electron beam position. Before fitting the timeresolved data at each pixel with the same function as for the average lifetime of Figure 2-b we binned four adjacent pixels to increase the signal to noise ratio. The equivalent integration time is therefore $20 \mathrm{~s}$ for each $12 \mathrm{~nm}$ width pixel. The lifetime extracted from the fit at each pixel is shown in Figure 3-c. The missing pixels represent pixels lying outside of the diamonds where the signal was too low to perform an accurate fit. Figure 3-d shows two decay traces acquired at different locations on the nano-diamonds and their corresponding fit. The measured lifetime depends strongly on the electron beam position and decreases from $23.4 \mathrm{~ns}$ to $15.8 \mathrm{~ns}$ when the electron beam is displaced by less than $50 \mathrm{~nm}$. The signal to noise ratio (SNR) of these spatially-resolved data is lower than in the case of Figure 2. We measured lifetime errors between 0.5 and $1 \mathrm{~ns}$ for most pixels and up to $2 \mathrm{~ns}$ for the noisiest pixel (See SI). Other examples of lifetime maps on nano-diamonds are given in the supplementary materials together with more details about the fitting procedure. The high brightness of our ultrafast electron source allows to generate a $1 \mathrm{~nm}$ electron probe ${ }^{37}$. However, it is important to stress the fact that the achievable spatial resolution will depend on the studied material and targeted temporal resolution. In particular, there is a compromise between the spatial resolution and signal-to-noise ratio that we can achieve. Indeed, optimizing the spatial resolution requires the use of small illumination apertures to remove the aberrations of the illumination system. The use of these apertures decreases the probe current and therefore the number of emitted photons. Therefore, even if our detection system is optimized (large collection angle, optimized coupling into the optical fiber and to the detection system), optimizing the spatial resolution might be too demanding in terms of signal to noise ratio in the case of weak emitters. Of course, adapting a probe aberration corrector would permit to keep the signal to noise ratio and achieve sub-nm electron beam size. The spatial resolution would therefore be only limited by the charge carrier diffusion length ${ }^{12}$. 

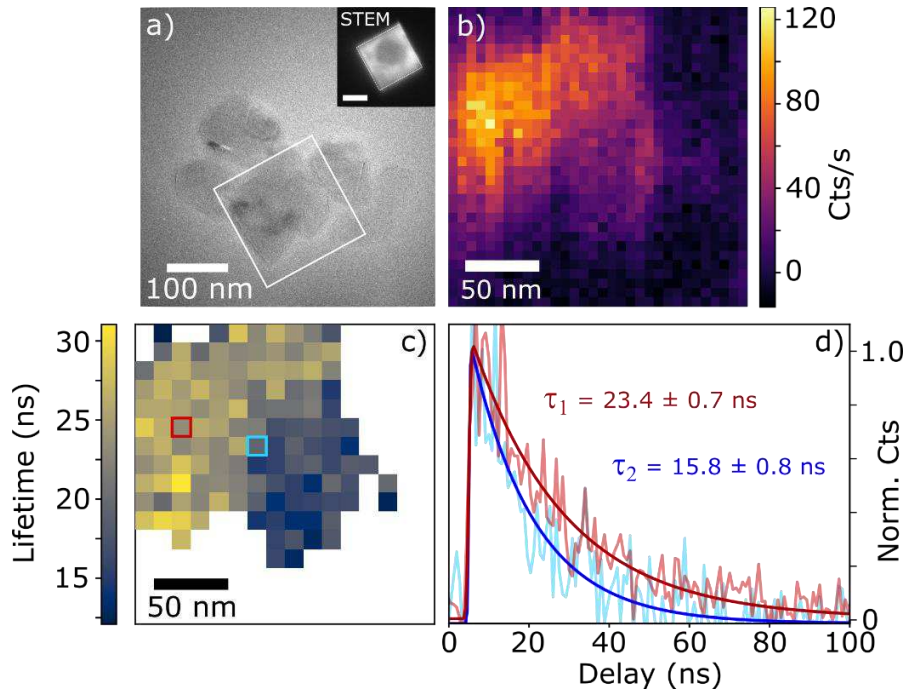

Figure 3 Mapping the spatial variations of the emission dynamics on a Nano-diamond cluster. a) TEM images of the cluster, the white square represents the scanned area. Inset: Scanning probe over the region of interest taken with the TEM CCD camera. scale bar $100 \mathrm{~nm}$ b) Intensity map extracted from the decay trace map by summing all the histogram bins of a $500 \mathrm{~ns}$ window, pixel size $6.3 \mathrm{~nm}$. c) Lifetime map after binning four adjacent pixels (effective pixel size $12.4 \mathrm{~nm}$ ). At each pixel a fit of the decay trace has been done and the extracted lifetime reported on the map. Pixels where the signal was too low to extract a lifetime are masked. D) Normalized decay traces and corresponding fit at the locations marked by the blue and red squares in c).

To conclude, we have performed time-resolved cathodoluminescence experiments in an Ultrafast Transmission Electron Microscope. Based on a high brightness cold-field emission source, this instrument can focus femtosecond electron pulses in a nanometric probe. The light from individual emitters is efficiently collected using a high numerical aperture parabolic mirror located close to the sample. This allowed us to map the lifetime of NV centers in nano-diamonds with a spatial resolution of about $12 \mathrm{~nm}$ and a sub-nanosecond temporal resolution. Such spatially-resolved lifetime measurements performed using an UTEM are extremely promising to investigate light emission dynamics from complex nanoscale systems ${ }^{20}$. In particular, the possibility of combining information on the emission dynamics together with structural, morphological, or chemical analysis at the atomic scale will bring invaluable information on the physics of the atomic scale light sources that play an increasing role in opto-electronic devices. Our study opens an application domain for Ultrafast Transmission Electron Microscopes and a wealth of possible experiments combining optical and electron excitation of nanoscale systems. The combination of a pulsed electron source and synchronized detection will allow coincidence experiments in which two or more complementary signals are detected (CL, diffraction, EELS, X-rays...) ${ }^{48}$. For instance, coincidence measurements of cathodoluminescence and EELS could give a unique insight in the quantum aspects of the electron/sample interaction ${ }^{49-51}$. Cathodoluminescence in a UTEM could be used to reveal the 
modification of the ultrafast response of a material resulting from the interference between excitations produced by light and free electrons ${ }^{52}$.

Supplementary Material:

Supplementary material contains a discussion on the lifetime map measurement error and the fit precision and showed other example of lifetime map on nanodiamonds.

Acknowledgment:

This project has been funded in part by the European Union's Horizon 2020 research and innovation program under grant agreement No 823717 (ESTEEM3) and 101017720 (EBEAM). This project has been funded in part by the ANR under the grant agreement ANR-19-CE30-0008 ECHOMELO and grant agreement ANR-14-CE26-0013 FemtoTEM.

Conflict of interest:

MK patented and licensed the technologies used in this work for light collection to Attolight for which he is a part time consultant at Attolight. All other authors declare no competing interests.

Data Availability Statement:

The data that support the findings of this study are available from the corresponding author upon reasonable request.

\section{References:}

1. Park K-D, Jeong H, Kim YH, et al. Time-Resolved Ultraviolet Near-Field Scanning Optical Microscope for Characterizing Photoluminescence Lifetime of Light-Emitting Devices. J Nanosci Nanotechnol. 2013;13(3).

2. Mintairov AM, Chu Y, He Y, et al. High-spatial-resolution near-field photoluminescence and imaging of whispering-gallery modes in semiconductor microdisks with embedded quantum dots. Phys Rev B - Condens Matter Mater Phys. 2008;77(19):1-7. doi:10.1103/PhysRevB.77.195322

3. Gaviola E. Ein Fluorometer. Apparat zur Messung von Fluoreszenzabklingungszeiten. Zeitschrift für Phys. 1927;42(11-12):853-861. doi:10.1007/BF01776683

4. Venetta BD. Microscope Phase Fluorometer for Determining the Fluorescence Lifetimes of Fluorochromes. Rev Sci Instrum. 1959;30(6):450-457. doi:10.1063/1.1716652

5. Lakowicz JR. Frequency-Domain Lifetime Measurements. In: Lakowicz JR, ed. Principles of Fluorescence Spectroscopy. Boston, MA: Springer US; 2006:157-204. doi:10.1007/978-0-38746312-4_5

6. Bugiel I, Konig K, Wabnitz $\mathrm{H}$. Investigation of cells by fluorescence laser scanning microscopy with subnanosecond time resolution. Lasers Life Sci. 1989;3(1):47-53.

7. Gruber A, Dräbenstedt A, Tietz C, Fleury L, Wrachtrup J, Von Borczyskowski C. Scanning confocal optical microscopy and magnetic resonance on single defect centers. Science (80-). 1997;276(5321):2012-2014. doi:10.1126/science.276.5321.2012 
8. Yacobi BG, Holt DB. Cathodoluminescence Microscopy of Inorganic Solids. New York: Springer; 1990.

9. Losquin A, Kociak M. Link between Cathodoluminescence and Electron Energy Loss Spectroscopy and the Radiative and Full Electromagnetic Local Density of States. ACS Photonics. 2015;2(11):1619-1627. doi:10.1021/acsphotonics.5b00416

10. Petroff PM, Lang D V. A new spectroscopic technique for imaging the spatial distribution of nonradiative defects in a scanning transmission electron microscope. Appl Phys Lett. 1977;31(2):60-62. doi:10.1063/1.89590

11. Yamamoto N, Araya K, García de Abajo F. Photon emission from silver particles induced by a highenergy electron beam. Phys Rev B. 2001;64(20):205419. doi:10.1103/PhysRevB.64.205419

12. Kociak M, Zagonel LF. Cathodoluminescence in the scanning transmission electron microscope. Ultramicroscopy. 2017;176:112-131. doi:https://doi.org/10.1016/j.ultramic.2017.03.014

13. Coenen T, Haegel NM. Cathodoluminescence for the 21st century: Learning more from light. Appl Phys Rev. 2017;4(3):031103. doi:10.1063/1.4985767

14. Zagonel LF, Mazzucco $S$, Tencé $M$, et al. Nanometer scale spectral imaging of quantum emitters in nanowires and its correlation to their atomically resolved structure. Nano Lett. 2011;11(2):568573. doi:10.1021/nl103549t

15. Griffiths JT, Zhang S, Rouet-Leduc B, et al. Nanocathodoluminescence Reveals Mitigation of the Stark Shift in InGaN Quantum Wells by Si Doping. Nano Lett. 2015;15(11):7639-7643. doi:10.1021/acs.nanolett.5b03531

16. Tizei LHG, Kociak M. Spectrally and spatially resolved cathodoluminescence of nanodiamonds: local variations of the NV(0) emission properties. Nanotechnology. 2012;23(17):175702. doi:10.1088/0957-4484/23/17/175702

17. Schmidt $G$, Berger $C$, Veit $P$, et al. Direct evidence of single quantum dot emission from $G a N$ islands formed at threading dislocations using nanoscale cathodoluminescence: A source of single photons in the ultraviolet. Appl Phys Lett. 2015;106(25). doi:10.1063/1.4922919

18. Meuret S, Tizei LHG, Auzelle T, et al. Lifetime Measurements Well below the Optical Diffraction Limit. ACS Photonics. 2016;3(7):1157-1163. doi:10.1021/acsphotonics.6b00212

19. Finot $\mathrm{S}$, Grenier V, Zubialevich V, et al. Carrier dynamics near a crack in GaN microwires with AlGaN multiple quantum wells. Appl Phys Lett. 2020;117(22):221105. doi:10.1063/5.0023545

20. Yanagimoto S, Yamamoto N, Sannomiya T, Akiba K. Purcell effect of nitrogen-vacancy centers in nanodiamond coupled to propagating and localized surface plasmons revealed by photoncorrelation cathodoluminescence. Phys Rev B. 2021;103(20):205418. doi:10.1103/PhysRevB.103.205418

21. Meuret $\mathrm{S}$, Solà Garcia $\mathrm{M}$, Coenen $\mathrm{T}$, et al. Complementary cathodoluminescence lifetime imaging configurations in a scanning electron microscope. Ultramicroscopy. 2019;197(November 2018):28-38. doi:10.1016/j.ultramic.2018.11.006

22. Christen J, Bimberg D. Cathodoluminescence Imaging of Semiconductor Interfaces. Oyo Buturi. 1988;57:69-77. 
23. Lin HT, Rich DH, Konkar A, Chen P, Madhukar A. Carrier relaxation and recombination in GaAs/AIGaAs quantum heterostructures and nanostructures probed with time-resolved cathodoluminescence. J App/ Phys. 1997;81(7):3186-3195. doi:10.1063/1.364148

24. Bell A, Christen J, Bertram F, et al. Localization versus field effects in single InGaN quantum wells Localization versus field effects in single InGaN quantum wells. Appl Phys Lett. 2004;84(58):1-4. doi:10.1063/1.1638880

25. Winkler D, Schmitt R, Brunner M, Lischke B. Flexible picosecond probing of integrated circuits with chopped electron beams. IBM J Res Dev. 1990;34(2.3):189-203. doi:10.1147/rd.342.0189

26. URA K, FUJIOKA H, HOSOKAWA T. Picosecond Pulse Stroboscopic Scanning Electron Microscope. J Electron Microsc (Tokyo). 1978;27(4):247-252. doi:10.1093/oxfordjournals.jmicro.a050126

27. Moerland RJ, Weppelman IGC, Garming MWH, Kruit P, Hoogenboom JP. Time-resolved cathodoluminescence microscopy with sub-nanosecond beam blanking for direct evaluation of the local density of states. Opt Express. 2016;24(21):24760. doi:10.1364/oe.24.024760

28. Merano $\mathrm{M}$, Sonderegger S, Crottini a, et al. Probing carrier dynamics in nanostructures by picosecond cathodoluminescence. Nature. 2005;438(7067):479-482. doi:10.1038/nature04298

29. Elsayed-Ali HE, Herman JW. Ultrahigh vacuum picosecond laser-driven electron diffraction system. Rev Sci Instrum. 1990;61(6):1636-1647. doi:10.1063/1.1141126

30. May P, Halbout J -M., Chiu G. Picosecond photoelectron scanning electron microscope for noncontact testing of integrated circuits. App/ Phys Lett. 1987;51(2):145-147. doi:10.1063/1.98596

31. Yang D, Mohammed OF, Zewail AH. Scanning ultrafast electron microscopy. 2010;2010. doi:10.1073/pnas.1009321107

32. Liu W, Mounir C, Rossbach G, et al. Spatially dependent carrier dynamics in single InGaN/GaN core-shell microrod by time-resolved cathodoluminescence. Appl Phys Lett. 2018;112(5):52106. doi:10.1063/1.5009728

33. Corfdir $P$, Lefebvre $P$, Balet $L$, et al. Exciton recombination dynamics in a -plane (Al,Ga)N/GaN quantum wells probed by picosecond photo and cathodoluminescence. J Appl Phys. 2010;107:43524. doi:10.1063/1.3305336

34. Fu X, Jacopin G, Shahmohammadi M, et al. Exciton Drift in Semiconductors under Uniform Strain Gradients: Application to Bent ZnO Microwires. ACS Nano. 2014;8(4):3412-3420.

35. Shahmohammadi M, Jacopin G, Fu X, Ganière J-D, Yu D, Deveaud B. Exciton hopping probed by picosecond time-resolved cathodoluminescence. Appl Phys Lett. 2015;107(14):141101. doi:10.1063/1.4932098

36. Houdellier F, Caruso GM, Weber S, Kociak M, Arbouet A. Development of a high brightness ultrafast Transmission Electron Microscope based on a laser-driven cold field emission source. Ultramicroscopy. 2018;186:128-138. doi:10.1016/j.ultramic.2017.12.015

37. Caruso GM, Houdellier F, Weber S, Kociak M, Arbouet A. High brightness ultrafast transmission electron microscope based on a laser-driven cold-field emission source: principle and applications. Adv Phys X. 2019;4(1):1660214. doi:10.1080/23746149.2019.1660214 
38. Caruso GM, Houdellier F, Abeilhou P, Arbouet A. Development of an ultrafast electron source based on a cold-field emission gun for ultrafast coherent TEM. Appl Phys Lett. 2017;111(2). doi:10.1063/1.4991681

39. Houdellier F, Caruso GM, Weber S, Hÿtch MJ, Gatel C, Arbouet A. Optimization of off-axis electron holography performed with femtosecond electron pulses. Ultramicroscopy. 2019;202:26-32. doi:https://doi.org/10.1016/j.ultramic.2019.03.016

40. Weber SJ. PyMoDAQ: An open-source Python-based software for modular data acquisition. Rev Sci Instrum. 2021;92(4):45104. doi:10.1063/5.0032116

41. Su L-J, Fang C-Y, Chang Y-T, et al. Creation of high density ensembles of nitrogen-vacancy centers in nitrogen-rich type lb nanodiamonds. Nanotechnology. 2013;24(31):315702. doi:10.1088/0957$4484 / 24 / 31 / 315702$

42. Botsoa J, Sauvage T, Adam M-P, et al. Optimal conditions for NV-center formation in type-1b diamond studied using photoluminescence and positron annihilation spectroscopies. Phys Rev $B$. 2011;84(12):125209. doi:10.1103/PhysRevB.84.125209

43. Schirhagl R, Chang K, Loretz M, Degen CL. Nitrogen-Vacancy Centers in Diamond: Nanoscale Sensors for Physics and Biology. Annu Rev Phys Chem. 2014;65(1):83-105. doi:10.1146/annurevphyschem-040513-103659

44. Mohtashami A, Femius Koenderink a. Suitability of nanodiamond nitrogen-vacancy centers for spontaneous emission control experiments. New J Phys. 2013;15(4):043017. doi:10.1088/13672630/15/4/043017

45. Solà-Garcia M, Meuret S, Coenen T, Polman A. Electron-induced state conversion in diamond NV centers measured with pump-probe cathodoluminescence spectroscopy. 2019;0:20-22. http://arxiv.org/abs/1910.04524.

46. Lourenço-Martins $\mathrm{H}$, Kociak M, Meuret $\mathrm{S}$, et al. Probing Plasmon-NVO Coupling at the Nanometer Scale with Photons and Fast Electrons. ACS Photonics. 2018;5(2):324-328. doi:10.1021/acsphotonics.7b01093

47. Lourenço-Martins $\mathrm{H}$, Kociak M, Meuret $\mathrm{S}$, et al. Probing Plasmon-NV 0 Coupling at the Nanometer Scale with Photons and Fast Electrons. ACS Photonics. 2017. doi:10.1021/acsphotonics.7b01093

48. Jannis D, Müller-Caspary K, Béché A, Oelsner A, Verbeeck J. Spectroscopic coincidence experiments in transmission electron microscopy. Appl Phys Lett. 2019;114(14):143101. doi:10.1063/1.5092945

49. Di Giulio V, Kociak M, de Abajo FJG. Probing quantum optical excitations with fast electrons. Optica. 2019;6(12):1524. doi:10.1364/optica.6.001524

50. Mechel C, Kurman Y, Karnieli A, Rivera N, Arie A, Kaminer I. Quantum correlations in electron microscopy. Optica. 2021;8(1):70. doi:10.1364/optica.402693

51. Kfir O, Giulio V Di, Javier García de Abajo F, Ropers C. Optical coherence transfer mediated by free electrons. Sci Adv. 2021;7(18):1-11. doi:10.1126/sciadv.abf6380

52. Di Giulio V, Kfir O, Ropers C, de Abajo FJ. Modulation of Cathodoluminescence Emission by Interference with External Light. ACS Nano. 2021;15(4):7290-7304. doi:10.1021/acsnano.1c00549 


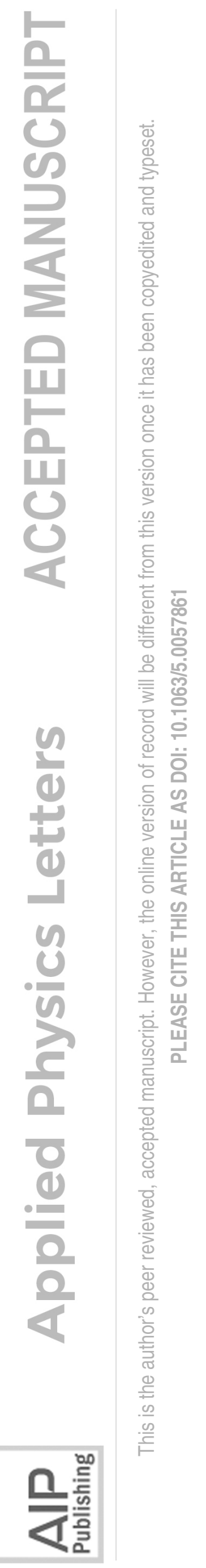




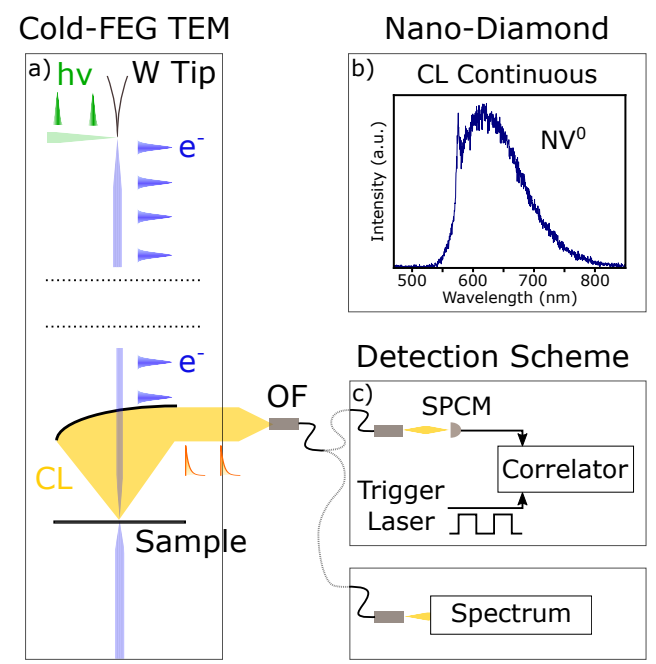

EELS, BF, Diff 


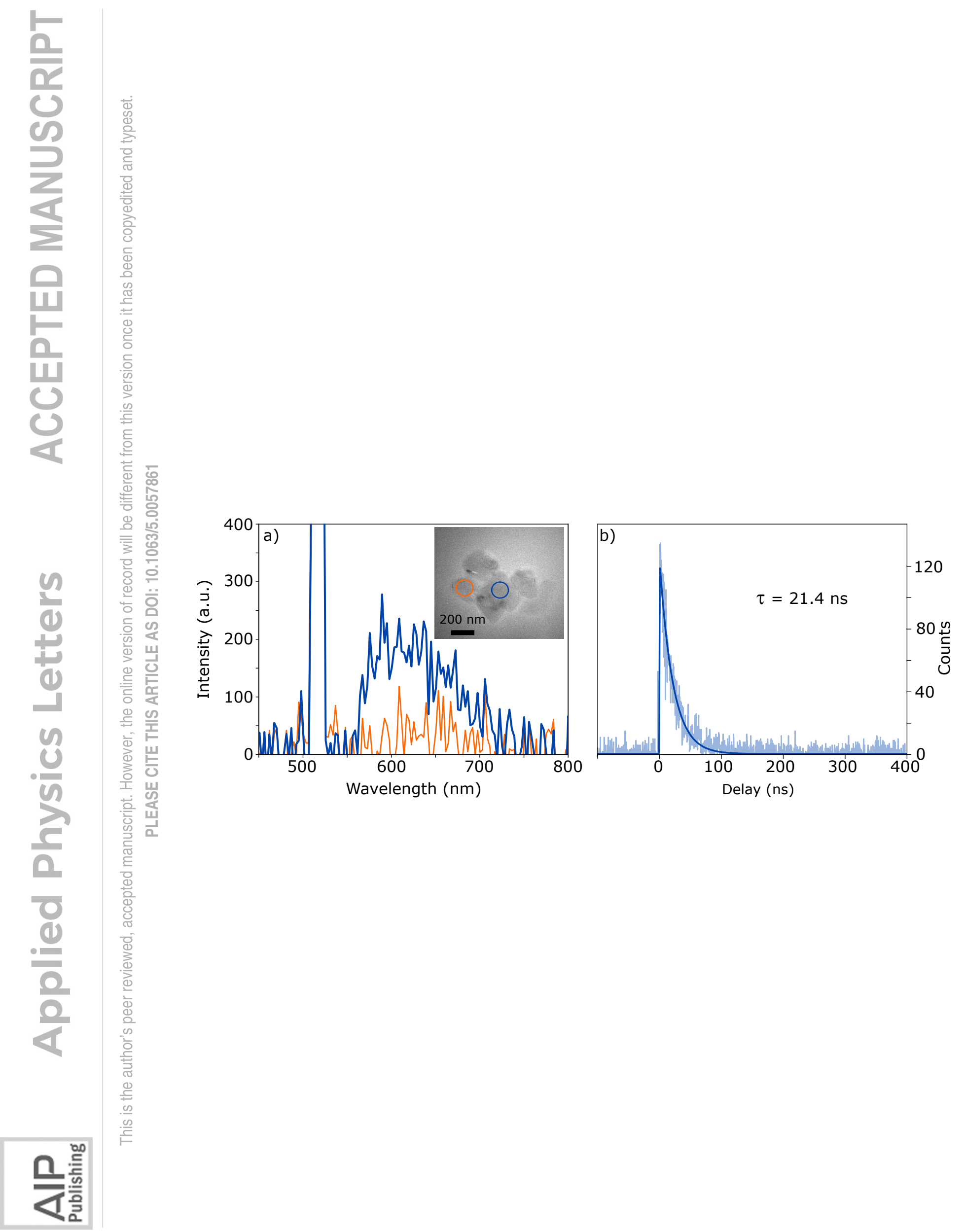



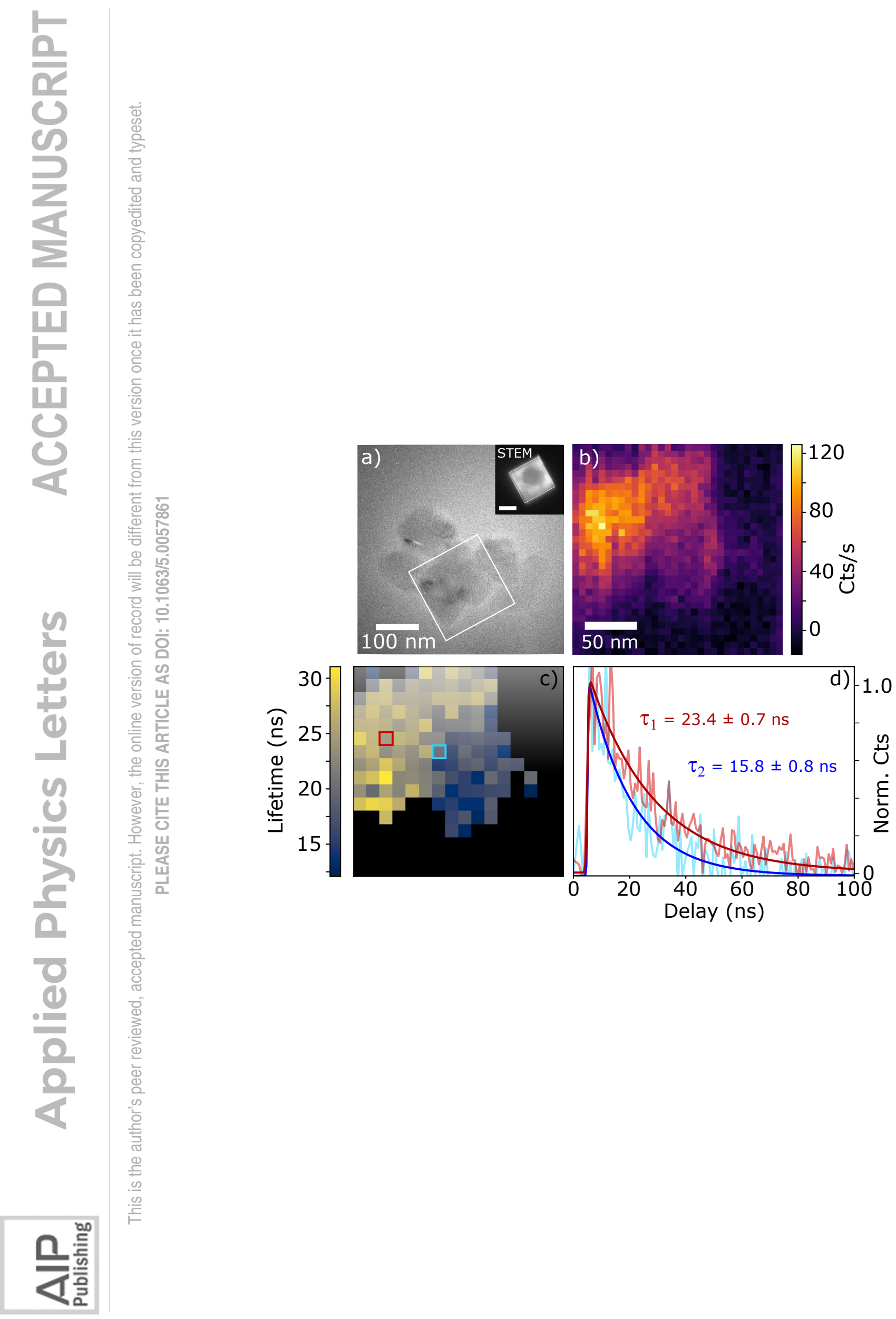